\title{
Spatiotemporal Measurement of Regional Expansion in Changsha-Zhuzhou-Xiangtan Metropolitan Area Based on Nighttime Light Data
}

\author{
Jian Zheng', Guoguang Wang ${ }^{1}$, Hua Wang ${ }^{2}$ \\ ${ }^{1}$ School of Architecture, State Key Laboratory of Subtropical Building Science, South China University of Technology, Guangzhou, \\ China \\ ${ }^{2}$ School of Architecture and Art, Central China University, Changsha, China \\ Email:2543930211@qq.com, arzhengjian@mail.scut.edu.cn,203126@csu.edu.cn
}

How to cite this paper: Zheng, J., Wang, G.G. and Wang, H. (2020) Spatiotemporal Measurement of Regional Expansion in Changsha-Zhuzhou-Xiangtan Metropolitan Area Based on Nighttime Light Data. Journal of Geographic Information System, 12, 202-220.

https://doi.org/10.4236/jgis.2020.123013

Received: May 18, 2020

Accepted: June 27, 2020

Published: June 30, 2020

Copyright ( 2020 by author(s) and Scientific Research Publishing Inc. This work is licensed under the Creative Commons Attribution International License (CC BY 4.0).

http://creativecommons.org/licenses/by/4.0/

(c) (i) Open Access

\begin{abstract}
The research purpose is to accurately reveal the temporal and spatial law of the urban expansion of Changsha-Zhuzhou-Xiangtan, one of the seven major urban agglomeration areas in China, and provide decision-making basis for the future urban construction land layout and regional development policy-making. Based on the night lighting data (DMSP/OLS), this paper extracts the boundary of the urban construction land of Changsha-Zhuzhou-Xiangtan urban agglomeration from 1993 to 2017, and quantitatively studies the spatial and temporal characteristics of the expansion of the metropolitan area in the past 25 years according to the methods of spatial expansion analysis, center of gravity migration measurement, landscape pattern index, spatial autocorrelation, etc. The results show that: 1) it is scientific and feasible to extract urban agglomeration construction land by the method of auxiliary data comparison for the study of urban expansion; 2) the expansion of regional space in Changsha-Zhuzhou-Xiangtan metropolitan area shows a trend of "weakening first and strengthening later". The construction land keeps increasing, and the expansion form gradually changes from extensive type to intensive type; 3 ) the center of gravity of the metropolitan area fluctuated and repeated in part during the past 25 years, but it was always located in the municipal district of Changsha city. The eastern region, mainly Changsha city, was still the core area of urban agglomeration expansion; 4) strengthening the territorial space protection and control of ecological green core in the metropolitan area is a key measure for the high-quality development of urban agglomeration.
\end{abstract}




\section{Keywords}

Changsha-Zhuzhou-Xiangtan, Spatial Expansion, Nighttime Light Data, Metropolitan Area, Control Scenario

\section{Introduction}

As the main carrier of carrying population and economic activities and supporting national economic growth, urban agglomeration is the main form of the country to promote new urbanization. Changsha-Zhuzhou-Xiangtan urban agglomeration is one of the seven urban intensive areas under the guidance and cultivation of the state, and one of the important urban agglomerations in the central region of China, which plays an important role in the national implementation of the medium and long-term regional development strategy [1]. Many scholars have studied the spatial structure and optimization measures of Changsha-Zhuzhou-Xiangtan urban agglomeration. However, the research results on the spatial and temporal law of regional expansion in metropolitan area are relatively rare [2] [3] [4].

In recent years, the development of remote sensing technology has provided new methods and approaches for the study of urban expansion. The DMSP/OLS night light images obtained by the operational line scan system (OLS) sensor on the US military meteorological satellite (DMSP) can be used as the characterization of human activities and a good data source for urbanization monitoring [5] [6]. At present, DSMP/OLS nighttime lighting data has been used to study the urban spatial expansion law at home and abroad, which fully proves the feasibility and scientificity of extracting urban spatial information [7] [8] [9] [10].

This paper selects the DSMP/OLS nighttime lighting data of ChangshaZhuzhou-Xiangtan urban agglomeration from 1993 to 2017, and uses the auxiliary data comparison method to extract the boundary of urban construction land through calculating the regional spatial expansion mode, landscape index and spatial autocorrelation index, quantitative study of the space-time law of metropolitan area, revealing the Changsha-Zhuzhou-Xiangtan urban agglomeration spatial and temporal process and characteristics in the past 25 years, in order for the city of the urban agglomeration development, to offer reference for policy making.

\section{Data Source and Processing}

\subsection{Overview of the Study Area}

The historical origin of the Changsha-Zhuzhou-Xiangtan urban agglomeration is the idea of "Mao Zedong City" proposed in 1954, and it is the earliest area for promoting integration and cooperation at the provincial level. In 1984, the "Changsha-Zhuzhou-Xiangtan Economic Zone" was proposed. In 1997, the "Changsha-Zhuzhou-Xiangtan Economic Integration" was officially proposed. 
In 2007, the state approved to establish Changsha-Zhuzhou-Xiangtan sustainable reform pilot zone, and officially entered the national central city from the economic zone era. In 2018, the "Changsha-Zhuzhou-Xiangtan Metropolitan Area" was proposed to promote high-quality development and to enter a new era of building a modern metropolitan area. The three cities of Changsha, Zhuzhou and Xiangtan, are distributed in the shape of "grade" along the Xiangjiang River. The distance between the two cities is less than 40 kilometers. They are in the hourly commuting circle, with both green belt isolation and high-speed road network connection (Figure 1). Changsha is the capital city of Hunan Province, the central strategic city of the Yangtze River in the strategic layout of the country, a relatively balanced industrial structure and high-level public services; Zhuzhou is an industrial star city represented by orbital equipment and an important transportation hub city; Xiangtan is an important industry, Science and education and tourism city. Changsha, Zhuzhou and Xiangtan has a population of 28,000 square kilometers, with a resident population of 15.4 million, GDP of 1579.6 billion yuan, respectively accounting for 13\%, 22\%, 43\% of Hunan Province, and Changsha-Zhuzhou-Xiangtan metropolitan area of 6528 square kilometers, accounting for $23 \%$ of the urban agglomeration. Basic economic statistics of the three cities in 2018: Changsha has a resident population of 8.15 million, GDP of 11.03 billion yuan, Zhuzhou resident population of 4.02 million, GDP of 263.2 billion yuan, Xiangtan resident population of 2.87 million, GDP of 216.1 billion yuan.

\subsection{Data Source and Processing}

1) Remote Sensing Data: The DMSP/OLS nighttime lighting data for this study was obtained from the National Geophysical Data Center website (http://ngdc.Noaa.gov). The acquired image has a width of $3000 \mathrm{~km}$, the ground

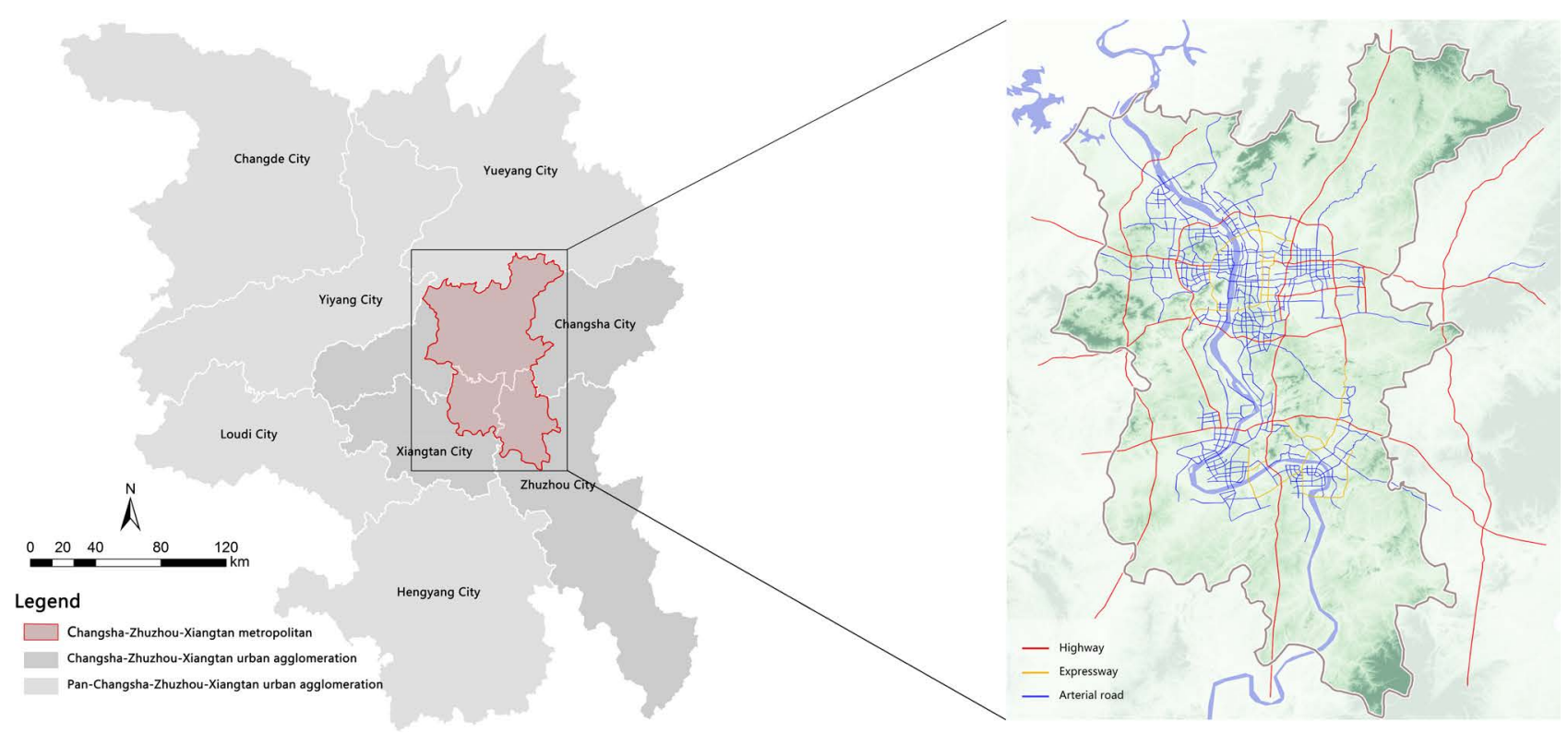

Figure 1. Location and scope map of the study area. 
sampling distance of the full resolution data is $0.56 \mathrm{~km}$, and the ground resolution is 0.0083333 degrees. The national nighttime light data obtained from 1993-2017 is the stable light image data. The data is raster grayscale remote sensing image, which is to directly average the gray value of the whole year VNIR channel after eliminating the effects of accidental noise such as clouds and flares. The data gray value range is $1-63$, the saturated light gray value is 63 , and the spatial resolution is $1 \mathrm{~km}$ [11]. At the same time, in order to improve the accuracy of space calculation, the research uses Landsat series satellite synchronization data to correct, and the sampling test accuracy, the data comes from the geospatial data cloud website (http://www.gscloud.cn/).

2) Statistics: The statistical aid data used in this study are mainly "the China Urban Statistical Yearbook" (1993-2017) and "the Hunan Statistical Yearbook" (1993-2017).

3) Auxiliary data: The county (district) and municipal level data in the national 1:4 million database are from the National Basic Geographic Information System website (http://ngcc.sbsm.gov.cn/). The nighttime light data of DMSP/OLS in different years in Changsha, Zhuzhou and Xiangtan area is shown in Figure 2.
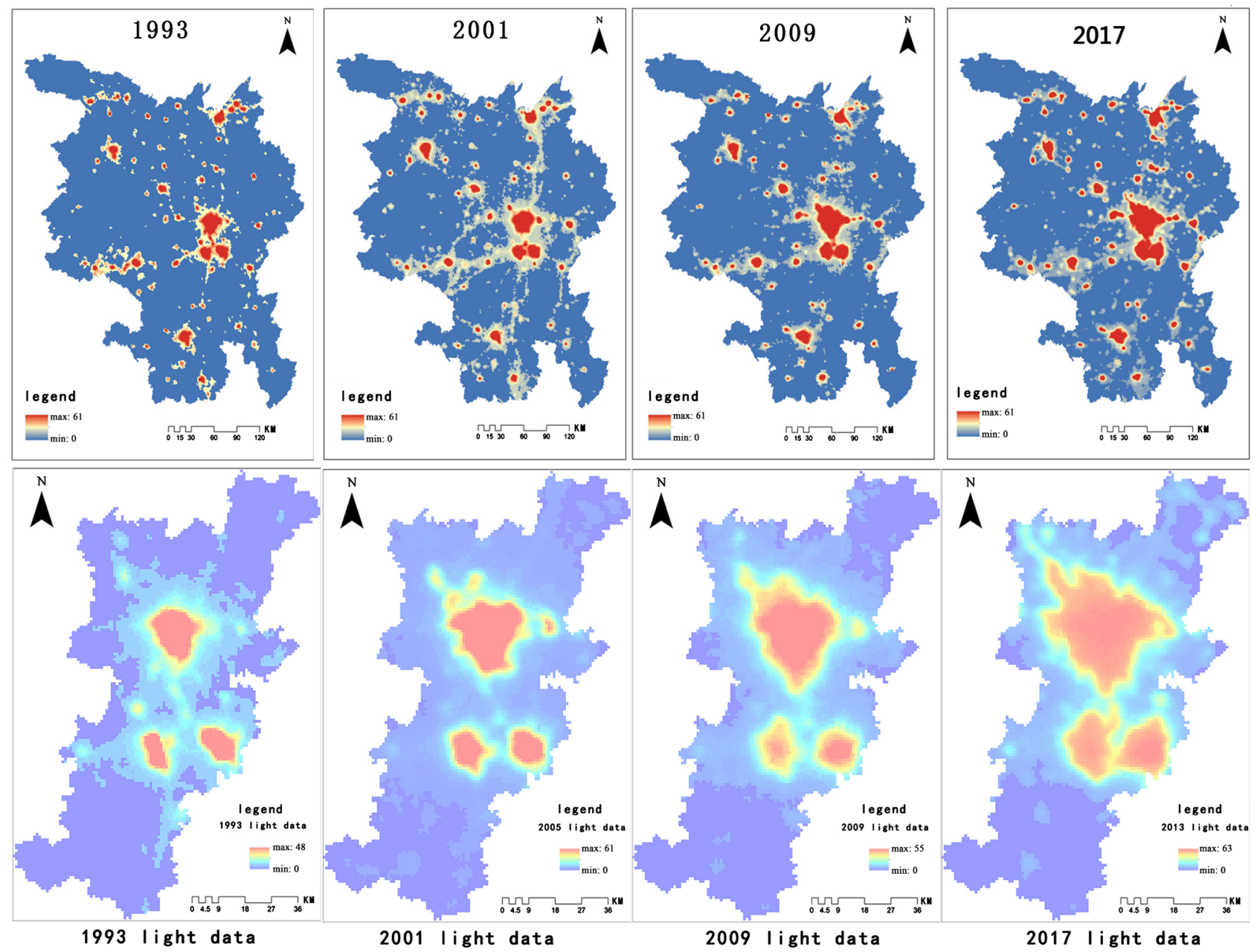

Figure 2. DMSP/OLS nighttime light data of different years in Changsha, Zhuzhou and Xiangtan. 


\section{Research Ideas and Methods}

\subsection{Light Threshold Method to Extract the Area of Urban Built-Up Area}

The extraction methods of urban built-up area mainly include empirical threshold method, mutation detection method, statistical data comparison method and high-resolution image data spatial comparison method. Reasonable determination of light threshold is the key to accurately extract the boundary of urban built-up area [12] [13]. In this paper, the auxiliary data comparison method is used, that is, the statistical data and high-resolution image are used as auxiliary data, the dichotomy method is used to determine the light threshold, the image self correction method (pixel by pixel algorithm) is used to denoise and correct the nighttime light data, and finally the Landsat $30 \mathrm{~m} \times 30 \mathrm{~m}$ high-resolution impact is used to verify the relative accuracy of extracted information. The research technology route is shown in Figure 3. In this study, each city in the urban agglomeration is treated as a whole. The corrected nighttime lighting data is used as the data source, and the dynamic threshold method is used to extract the boundary of the urban construction land of the Changsha-Zhuzhou-Xiangtan

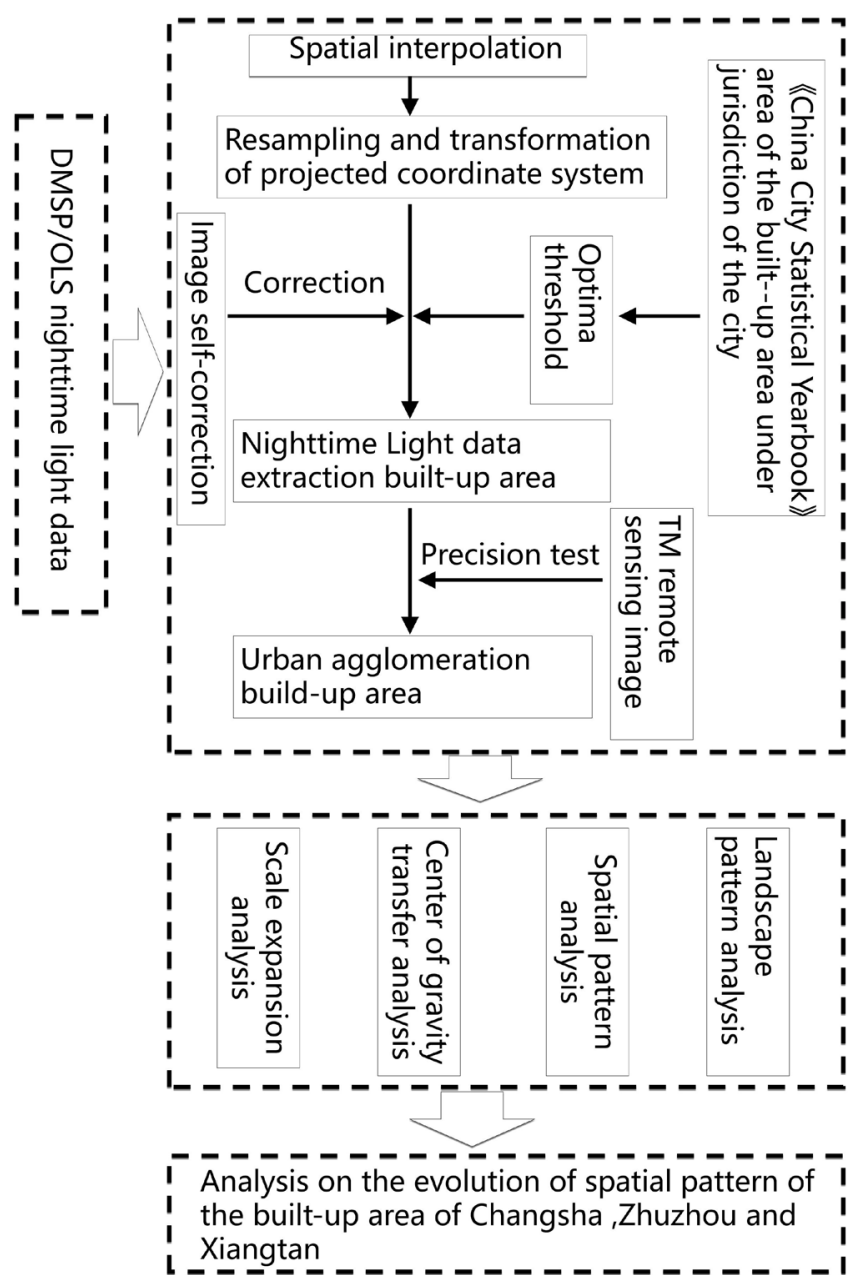

Figure 3. Research technology roadmap. 
urban agglomeration, when the dynamic threshold is under the light. The threshold value when the absolute difference between the area and the built-up area of the municipal district is the smallest is taken as the optimal threshold, and the area of the light larger than the threshold is the built-up area. According to the optimal threshold, the scope of urban construction land in Changsha, Zhuzhou and Xiangtan urban agglomerations is extracted [14] [15]. The nighttime light data of DMSP/OLS in different years in Changsha, Zhuzhou and Xiangtan area is shown in Figure 2.

\subsection{Accuracy Test}

The $30 \mathrm{~m} \times 30 \mathrm{~m}$ high-resolution Landsat Image in the local area of Changsha-Zhuzhou-Xiangtan urban agglomeration is selected as the verification data to evaluate the relative accuracy of the information extracted from the auxiliary data comparison method. According to the area covered by the satellite film, 100 sample points were randomly selected from the built-up area and the non built-up area in 2015. Then use the TM image of the region in 2015 as the validation data to evaluate the accuracy of the built-up area in 2015. The accuracy evaluation results are shown in Table 1 . The producer accuracy and user accuracy are both above $80 \%$, and the kappa coefficient is 0.7429 . It can be seen that the accuracy of the information extracted by the statistical data comparison method is relatively high, which indicates that the urban information extracted in this paper can be used to study the dynamic analysis of land use expansion in Changsha-Zhuzhou-Xiangtan urban agglomeration.

\subsection{Analysis of Spatial Scale Expansion of Metropolitan Area}

Based on the nighttime light data of different years, the scope of urban agglomeration built-up areas in different years can be extracted, and then the scale expansion of built-up areas can be studied year by year. The analysis of scale expansion mainly includes expansion mode, expansion speed, expansion intensity and other indicators. The urban spatial expansion model is based on the type of urban spatial expansion evolution process [16]. The expansion speed is the absolute increment of the expansion area of the urban agglomeration built-up areas at different stages, representing the overall scale and trend of urban built-up area expansion [17].

Table 1. Sampling accuracy test confusion matrix (2015).

\begin{tabular}{ccccc}
\hline type & Urban land use & Non-urban land & sum & User accuracy \\
\hline Urban land $\left(\mathrm{m}^{2}\right)$ & $39,783,032.45$ & $8,123,754.395$ & $47,906,786.84$ & $83.04 \%$ \\
Non-urban land $\left(\mathrm{m}^{2}\right)$ & $9,054,021.57$ & $99,463,290.56$ & $108,517,312.1$ & $91.66 \%$ \\
sum $\left(\mathrm{m}^{2}\right)$ & $48,837,054.02$ & $107,587,045$ & $156,424,099$ & \\
Producer accuracy & & & & $92.45 \%$ \\
\hline
\end{tabular}

Kappa Coefficient $=0.7429$. 


$$
\mathrm{A}=\frac{\Delta S}{\Delta t \times S} \times 100 \%
$$

In the formula, $A$ is the expansion speed of the built-up area; $S$ is the total area of the urban built-up area at the beginning of the study; $\Delta S$ is the extended area of the urban built-up area; $\Delta t$ is the time interval, and the larger $A$ is, the faster the expansion rate of the built-up area is.

The expansion intensity is the ratio of the expanded area of the urban agglomeration built-up area to the total area of the urban agglomeration in a certain period of time. Since the expansion speed cannot quantitatively compare the degree of urban expansion, the intensity of urban expansion is introduced to reflect the strength and speed of urban expansion, making the speed of urban expansion in different periods comparable.

$$
\mathrm{I}=\frac{\Delta U}{\Delta t \times T A} \times 100 \%
$$

In the formula, $I$ represents the urban expansion intensity index; $T A$ is the total land area of the urban agglomeration, and $\Delta U$ is the area difference extended in different periods. The larger $I$ is, the greater the intensity of urban expansion.

\subsection{Metropolis Area Center of Gravity Determination}

Changsha-Zhuzhou-Xiangtan metropolis area center of gravity $(X, Y)$ is defined as the average center position of all built-up areas in the study area. The change of the center of gravity of the built-up area can reflect the change of the spatial distribution of the built-up area. The built-up area of the urban agglomeration extracted by this study is composed of pixels with different gray values. The center coordinates of the I pixel in the $t$ year are $\left(X_{t i}, Y_{t i}\right)$ and $M_{t i}$ is the gray value of the pixel in the t year, where $X_{t}$ and $Y_{t}$ represent the longitude and latitude coordinates of the center of gravity of the urban agglomeration in the $t$ year. Then the coordinates of the center of gravity of the built-up area weighted by gray values are:

$$
X_{t}=\frac{\sum_{i=1}^{n} M_{t i} X_{t i}}{\sum_{i=1}^{n} M_{t i}}, Y_{t}=\frac{\sum_{i=1}^{n} M_{t i} Y_{t i}}{\sum_{i=1}^{n} M_{t i}}
$$

The center of gravity offset distance $\Delta t$, refers to the moving distance of the center of gravity of the built-up area of the urban agglomeration during a certain research period. The average migration velocity $V t$ refers to the average movement velocity of the center of gravity of the urban agglomeration in a certain research period, which is used to visually reflect the spatial difference of the expansion of the built-up areas of the urban agglomeration. The offset angle $\alpha_{t}$ refers to the angle between the direction in which the center of gravity of the metropolis area moves and the east in a certain research period.

$$
\begin{gathered}
\Delta_{t}=\sqrt{\left(X_{t_{i+1}}-X_{t_{i}}\right)^{2}+\left(Y_{t_{i+1}}-Y_{i}\right)^{2}} \\
V_{t_{i+1}-t_{i}}=\frac{\Delta_{t}}{t_{i+1}-t_{i}}
\end{gathered}
$$




$$
\alpha_{t}=n \pi+\arctan \frac{y_{t_{i+1}}-y_{t_{i}}}{x_{t_{i+1}}-x_{t_{i}}} \arctan \frac{y_{t_{i+1}}-y_{t_{i}}}{x_{t_{i+1}}-x_{t_{i}}},(n=0,1,2)
$$

In the formula, $X_{t}$ and $Y_{t}$ are the center of gravity coordinates of the construction land within a certain period, $t_{i+1}-t_{i}$ represents the time span of center of gravity shift in the built-up area; $V$ represents the annual migration rate of the center of gravity in the built-up area within the time span of $t_{i+1}-t_{i}$.

\subsection{Analysis on the Evolution of Metropolitan Landscape Pattern}

The main research object of landscape ecology is the landscape pattern. The study of landscape pattern is mainly realized by the landscape index. The landscape index is a simple quantitative index that can highly enrich the landscape pattern information and reflect some aspects of its structural composition and spatial configuration. Based on the basic characteristics of Changsha-ZhuzhouXiangtan metropolise expansion, this study selects landscape level index and patch type level index in landscape index to study the evolution of spatial pattern in built-up areas of Changsha-Zhuzhou-Xiangtan urban agglomeration. These indexes were selected such as the number of patches (NP), Plaque density (PD), largest plaque index (LPI), Landscape shape index (LSI), connectivity index (COHESION), aggregation index (AI), and Shannon diversity index (SHDI) [18]. The largest patches index (LPI) is used to represent the primacy in the urban agglomeration region. Connectivity index (COHESION) reflects the natural connectivity of patch types, which can reflect the natural connectivity of patches under different distance thresholds. Aggregation index (AI) is calculated based on the length of the common edges between the pixels of the same type of patch. The degree of aggregation reflects the degree of spatial aggregation of patches of landscape type. The Shannon diversity index (SHDI) is used to indicate the degree of land use richness and fragmentation [h8].

\subsection{Spatial Autocorrelation Analysis}

After extracting the spatial information of Changsha-Zhuzhou-Xiangtan urban agglomeration over the years using night light data, in order to comprehensively measure the agglomeration or diffusion characteristics of urban agglomeration spatial expansion, Moran's I index, a spatial autocorrelation model commonly used in econometric geography, is introduced. Its calculation method is as follows:

$$
\begin{aligned}
\text { Moran's I } & =\frac{\sum_{i=1}^{n} \sum_{j=1}^{n} W_{i j}\left(Y_{i}-\bar{Y}\right)\left(Y_{j}-\bar{Y}\right)}{S^{2} \sum_{i=1}^{n} \sum_{i=1}^{n} W_{i j}} \\
S^{2} & =\frac{1}{n} \sum_{i=1}^{n}\left(Y_{i}-\bar{Y}\right), \bar{Y}=\sum_{i=1}^{n} Y_{i}
\end{aligned}
$$

In the formula, $\mathrm{n}$ represents the number of cities in each district and county of Changsha-Zhuzhou-Xiangtan urban agglomeration; Yi indicates the $i$-th urban space expansion area; $W_{i j}$ represents the spatial adjacency of research units. $W_{i}$ 
takes 1 when two research area units are adjacent. when two research area units are not adjacent, the value of $W_{i j}$ is 0 . Moran's has a value range of $-1 \leq I \leq 1$. The closer I value is to 1 , the agglomeration trend of urban agglomeration spatial growth is shown. The closer I value is to -1 , the discrete state of urban agglomeration spatial growth is shown.

\section{Results and Analysis}

\subsection{Analysis on the Process of Metropolis Expansion}

In this study, the area information of urban built-up areas (municipal districts) in urban agglomeration in the statistical yearbook and DMSP/OLS nighttime lighting data are used to obtain the best threshold value of built-up areas in different periods of each city though threshold dichotomy. The average error values between the area of built-up areas extracted from light data and the data in the China Urban Statistical Yearbook are 1.64\%, $-0.10 \%, 2.27 \%, 0.54 \%,-0.85 \%$, $0.27 \%, 0.05 \%,-0.22 \%, 0.50 \%, 1.19 \%,-0.88 \%, 1.26 \%$, all controlled within $3 \%$. The error of each city is basically controlled within $5 \%$ and the overall accuracy of the data is relatively high, and spatial information from 1993 to 2017(total 12 years) is extracted respectively according to the optimal threshold value of each city. The extracted built-up areas are $818.29 \mathrm{~km}^{2}, 946.58 \mathrm{~km}^{2}, 1037.66 \mathrm{~km}^{2}$, $1097.20 \mathrm{~km}^{2}, 1363.41 \mathrm{~km}^{2}, 1739.25 \mathrm{~km}^{2}, 2119.69 \mathrm{~km}^{2}, 2273.46 \mathrm{~km}^{2}, 2333.13 \mathrm{~km}^{2}$, $2570.78 \mathrm{~km}^{2}, 2825.40 \mathrm{~km}^{2}$ and $3225.46 \mathrm{~km}^{2}$. According to the statistical table of time scale expansion analysis of the built-up area (Figure 4), the expansion area, expansion speed and expansion intensity of the urban agglomeration showed a W-shaped development trend from 1993 to 2017. The three indicators decreased continuously from 1993 to 1999. The expansion area and expansion speed increased rapidly from 1999 to 2005 and reached the maximum value in 2005, which were $380.44 \mathrm{~km}^{2}$ and $190.22 \mathrm{~km}^{2}$ respectively. Then the expansion speed and expansion intensity decreased continuously from 2005 to 2009 and began to

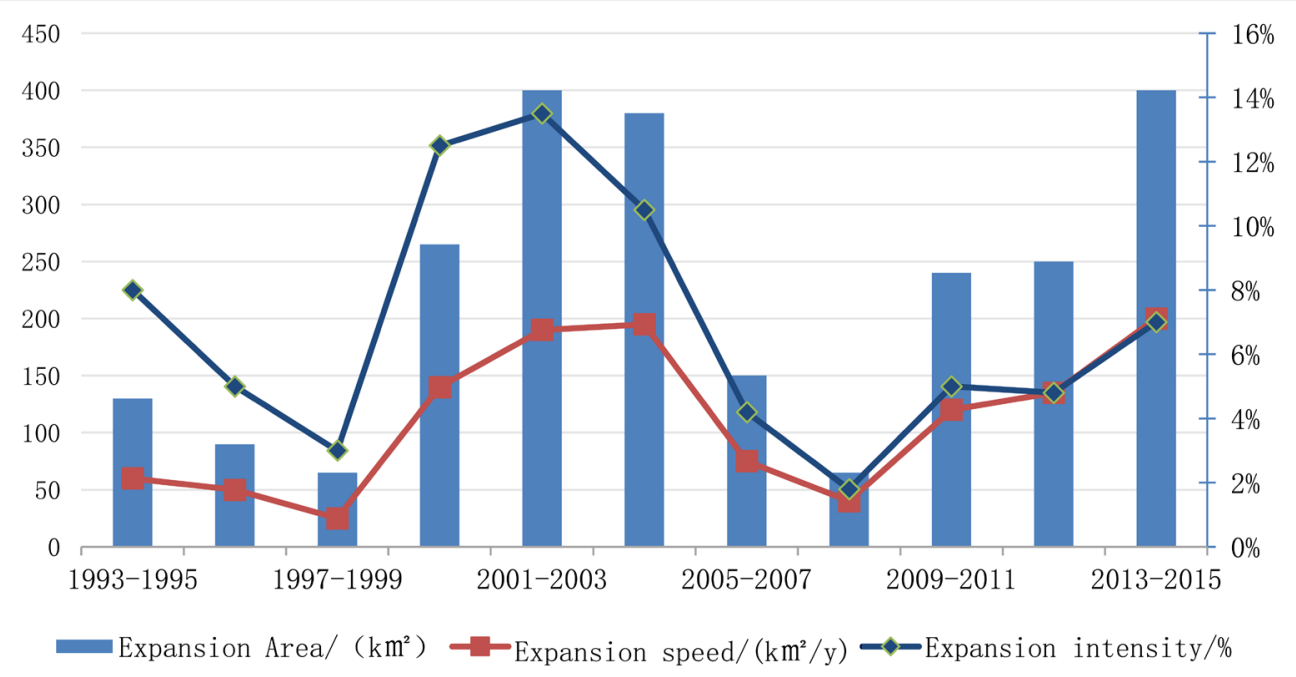

Figure 4. Statistical chart of time scale expansion analysis of built-up area. 
increase slowly from 2009 to 2015 . The expansion intensity increased rapidly from 1999 to 2003 , reaching a maximum of $13.78 \%$ in 2003. It decreased rapidly in 2003-2009 and began to increase slowly again in 2009-2017. In general, the expansion area of metropolitan area has been continuously increasing, and gradually changed from extensive expansion to intensive expansion.

\subsection{Urban Expansion Model}

According to the situation of land use expansion in Figure 5, it can be seen that

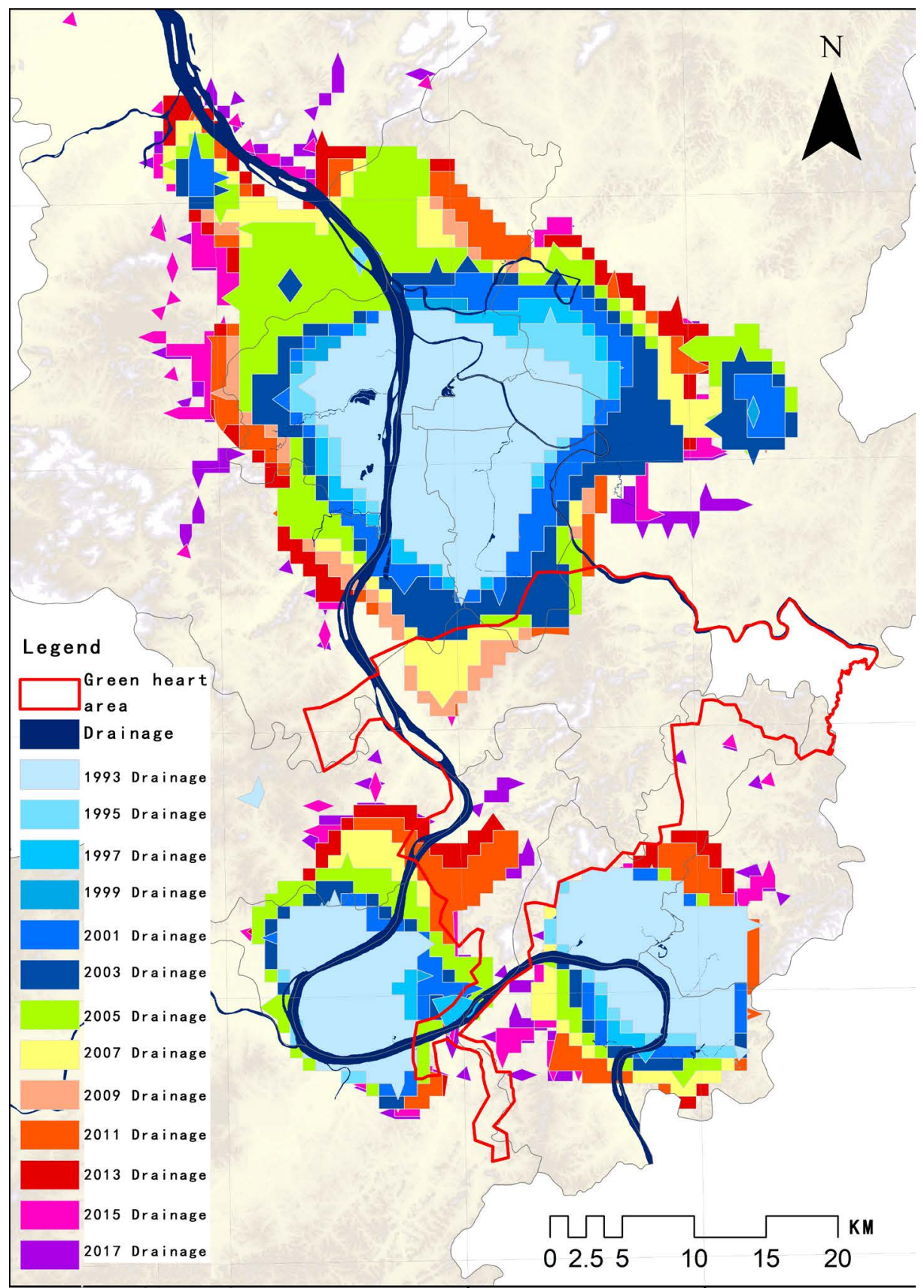

Figure 5. Schematic diagram of construction land expansion in Changsha-Zhuzhou-Xiangtan urban. 
the main urban areas of Changsha-Zhuzhou-Xiangtan urban agglomeration are still in a planar development mode, with irregular circles expanding outward. The growth of small towns in the periphery of big cities shows a dot pattern, and some areas show linear development along with the aid of transportation, such as Ningxiang, Xiangxiang, Liuyang and Linning. The integration trend of the three cities of Changsha, Zhuzhou and Xiangtan is obvious. In addition to the integration of Zhuzhou and Xiangtan to the south, Changsha also expands to the northwest. Zhuzhou and Xiangtan are mainly developing towards Changsha, and they are also close to each other.

\subsection{The Dominant Direction of the Metropolises Expansion}

According to the extracted patches of urban construction land in each city group from the urban agglomeration, the gray value of pixels in the patches is taken as the weight, and the higher the gray value of pixels, the richer the light intensity information is, and the more active the city can be reflected. According to the formula (3), the longitude and latitude coordinates of the center of gravity of Changsha-Zhuzhou-Xiangtan urban agglomeration over the years are calculated. And reconstruct the center of gravity model of the Changsha-Zhuzhou-Xiangtan urban agglomeration, and invert the trajectory of the center of gravity of the Changsha-Zhuzhou-Xiangtan metropolis area from 1993 to 2017 (Figure 6).

Figure 6 shows that the center of gravity of Changsha-Zhuzhou-Xiangtan metropolis area from 1993 to 2017 is located in Changsha city, and the center of gravity from 1993 to 1999 is located in Yuelu District. From 1999 to 2015, the center of gravity fell in Wangcheng District. It can be seen from the position of the center of gravity that the urbanization level of the central and eastern regions of the Changsha-Zhuzhou-Xiangtan urban agglomeration is higher than that of the western regions. And during this 25 -year shift in the center of gravity, local

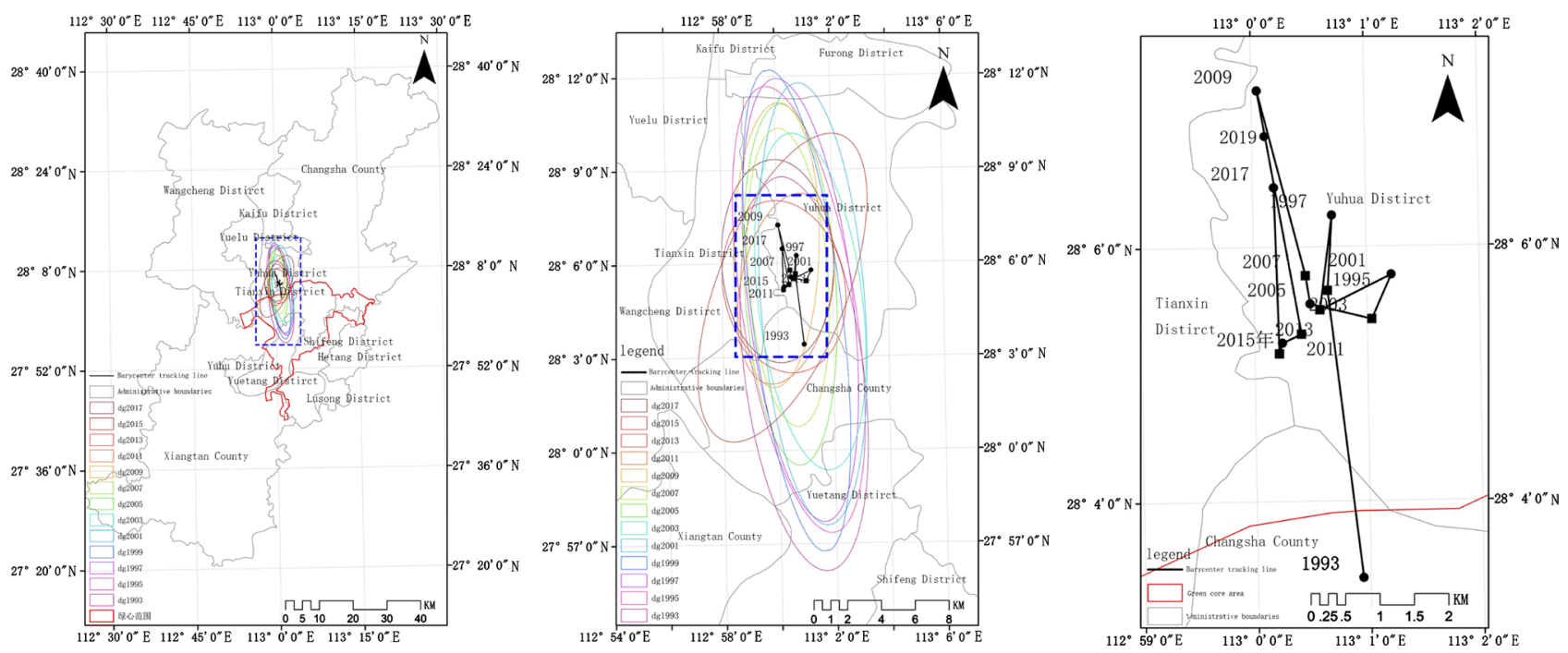

Figure 6. Ellipse of standard deviation of urban scale and change trend of center of gravity for construction land in Changsha-Zhuzhou-Xiangtan metropolitan area. 
fluctuations and iterations have occurred, and the center of gravity has changed dramatically in some years. In 1993-1995, the center of gravity moved to the northwest, and the movement speed was the fastest, reaching $5342.41 \mathrm{~m} /$ year. From 1995 to 1997, it turned to the southeast and continued to move to the west from 1997 to 2003 . In 2001 to 2003 , the center of gravity rapidly moved to the south, with an offset distance of 10,438.88 meters and an offset angle of-95.83. For 8 consecutive years from 2003 to 2011, the center of gravity turned back to the east, and the overall trend was toward the southeast. From 2011 to 2013, the center of gravity experienced another sharp fluctuation, shifting 10,265.13 meters from southeast to northeast with an angle of 126.09. In 2013-2015, it turned to the southeast again. During the past 25 years, the overall trend of the center of gravity of the metropolis has moved toward the northwest as a whole, then to the southeast, and finally to the northwest (Table 2).

\subsection{Landscape Morphological Characteristics of Metropolitan Area}

The landscape pattern analysis software Fragstats 4.2 is used to quantitatively calculate the landscape pattern of the regional expansion of Changsha-ZhuzhouXiangtan urban agglomeration, and the change trend map of each index of the landscape pattern of Changsha-Zhuzhou-Xiangtan urban agglomeration from 1993 to 2017 is obtained. The total number of patches (NP) and the landscape shape index (LSI) can both represent the degree of spread of urban agglomeration built-up areas. Both showed an overall trend of increasing first and then decreasing. In 2005, both reached a maximum. The maximum number of patches (NP) was 114, and the maximum value of the landscape shape index (LSI) was 24.79(Figure 7).

The total number of patches (NP) indicates the expansion of urban land use.

Table 2. Track of Center of Gravity Shift in Changsha-Zhuzhou-Xiangtan metropolitan area.

\begin{tabular}{cccc}
\hline Time & Offset distance $/ \mathrm{m}$ & Offset speed $/(\mathrm{m} / \mathrm{s})$ & Offset angle $^{\bullet}$ \\
\hline $1993-1995$ & $10,864.82$ & 5432.41 & 121.81 \\
$1995-1997$ & 5458.41 & 2729.20 & -29.17 \\
$1997-1999$ & 7373.87 & 3686.94 & -153.75 \\
$1999-2001$ & 6723.05 & 3361.53 & 114.45 \\
$2001-2003$ & $10,438.88$ & 5219.44 & -95.83 \\
$2003-2005$ & 3772.90 & 1886.45 & 66.13 \\
$2005-2007$ & 1332.19 & 666.10 & 24.04 \\
$2007-2009$ & 6873.97 & 3436.99 & -59.40 \\
$2009-2011$ & 1847.86 & 923.93 & -86.29 \\
$2011-2013$ & $10,265.13$ & 5132.56 & 126.09 \\
$2013-2017$ & 4424.81 & 2212.40 & -62.80 \\
\hline
\end{tabular}


NP increased slowly in 1993-1999 and increased rapidly in 1999-2005. During this period, the total number of patches increased by 5.3 per year, and then decreased by 2.8 per year from 2005 to 2015. Landscape shape index (LSI) indicates the complexity of patch shape. The overall increase of LSI in 1993-2005 indicates that the shape of urban construction land is constantly complicated, and the overall reduction in 2005-2015 indicates that the shape of urban construction land is more and more regular (Figure 7). The two indexes meet the general rule of urban spatial expansion. At the initial stage of urban expansion, the growth point of cities and towns does not expand continuously, the number of patches increases, and the degree of fragmentation becomes higher and higher. With the passage of time, cities and towns continue to expand, independent urban patches tend to be connected into patches, the number of patches is reduced, the complexity is continuously reduced, and the development of cities and towns is more compact.

The connectivity index (COHESION) and aggregation index (AI) showed an overall increasing trend from 1993 to 2015, and the rate of change gradually increased, reaching the maximum in 2015, 68.63 and 42.26 respectively. It indicates that the urban area of Changsha-Zhuzhou-Xiangtan is expanding, the distribution of urban landscape is centralizing, and the degree of integration is improving (Figure 7). The patch density (PD) also shows the same development law. It has been increasing from 1993 to 2015, reaching the maximum value of 0.72 in 2015 (Figure 8).
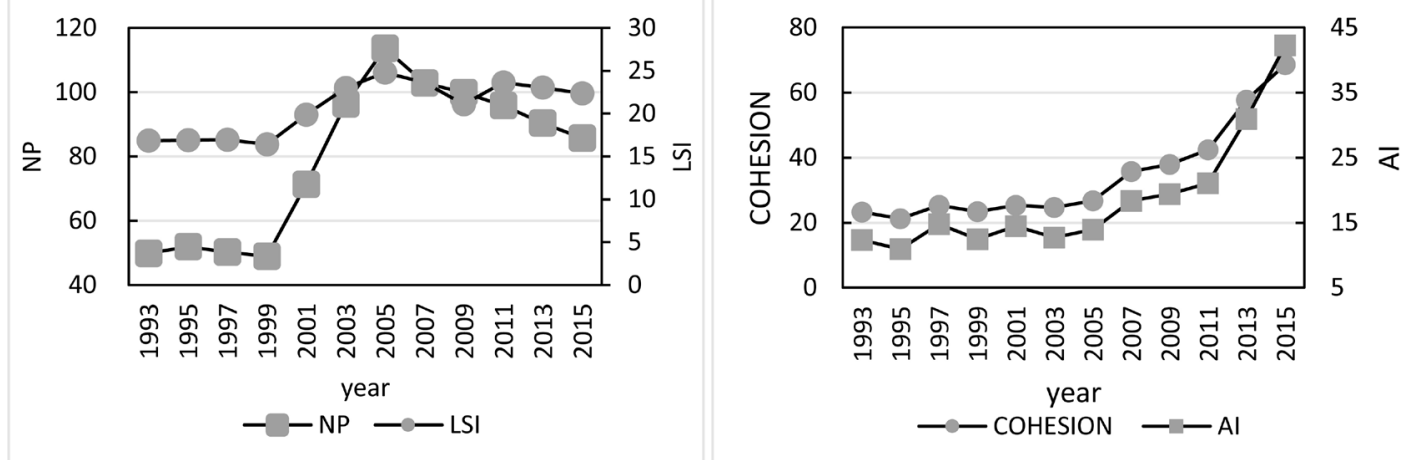

Figure 7. NP, LSI, COHESION, AI Index.
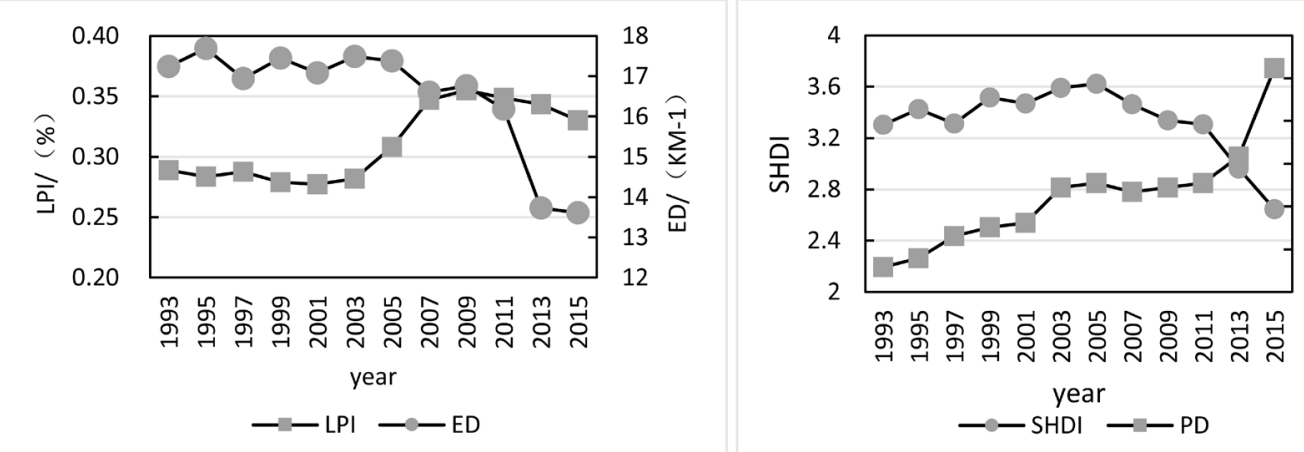

Figure 8. LPI, ED, SHDI, PD index. 
The average edge density (ED) and the Shannon diversity index (SHDI) of the Changsha-Zhuzhou-Xiangtan urban agglomeration increased slightly between 1993 and 2005, and declined in 2005-2015. The Shannon diversity index (SHDI) is used to indicate the degree of land use richness and fragmentation, and its change trend is consistent with the above analysis results (Figure 8).

The largest patches index(LPI) declined slightly from 1993 to 2003, then increased continuously, reaching the maximum value of 0.355 in 2009 and decreased continuously from 2009 to 2015(Figure 8). It indicates that Changsha City has played an important role in leading and radiating the surrounding areas in the development of the urban agglomeration, and has driven the development of the urban agglomeration to be more balanced in the process of its continuous development.

\subsection{The Trend of Metropolis Spatial Expansion}

According to the nighttime light data, calculate the Moran's I index and $\mathrm{Z}$ value respectively from 1995 to 2017 with formula (5). The results are shown in Figure 9. As a whole, it can be seen that $Z$ value and Moran's I show the trend of first decreasing and then increasing. From 1995 to 2003, Moran's I index decreased from 0.6038 to 0.2771 , with some fluctuations in $\mathrm{Z}$ value, which increased first and then decreased in a small range; while from 2003 to 2015, Moran's I index and $\mathrm{Z}$ value increased rapidly, from 0.2771 and 8.5333 to 0.5995 and 18.3410, respectively. It shows that the spatial expansion of Changsha-Zhuzhou-Xiangtan urban agglomeration shows a trend of continuous "cohesion", which gradually weakened from 1995 to 2003, and accelerated from 2003 to 2015. In general, it shows a trend of "weakening first and then strengthening".

\section{Changsha-Zhuzhou-Xiangtan Metropolis Area Spatial Management and Control}

At present, the construction land of the Changsha, Zhuzhou and Xiangtan

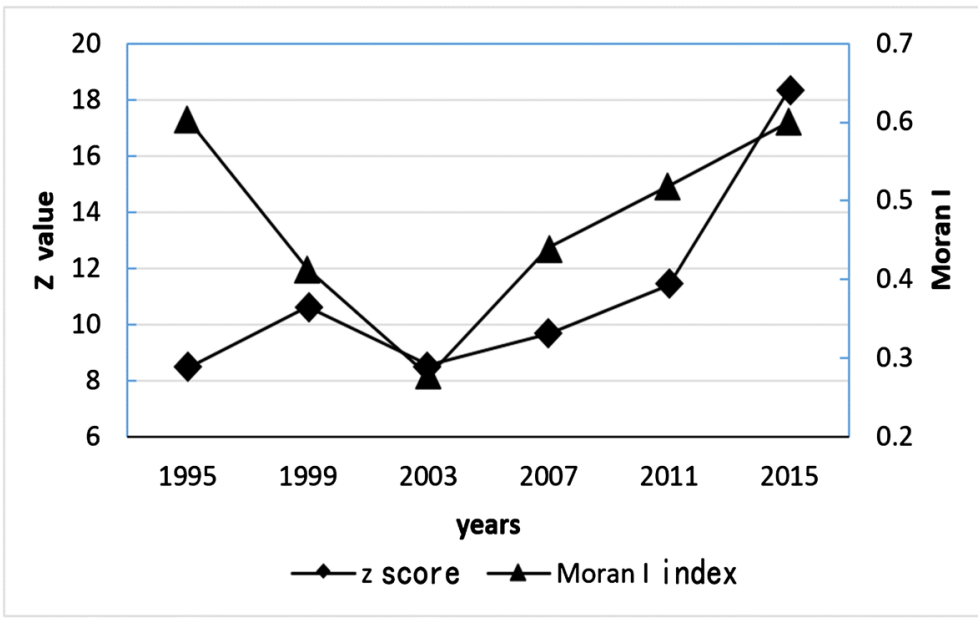

Figure 9. Changsha-Zhuzhou-Xiangtan urban agglomeration Moran's I index and Z value trend chart. 
metropolitan areas has been partially blended, and the ecological green heart of the Changsha, Zhuzhou and Xiangtan metropolitan areas has been gradually eroded. According to the characteristics of construction land expansion of the Changsha-Zhuzhou-City urban area in 1993-2017, with reference to the overall planning of the three cities of Changsha, Zhuzhou and Xiangtan and related research results [19] [20] [21], choose three different control scenarios: no control, general control and strict control. Forecast on the future development of construction land in Changsha, Zhuzhou and Xiangtan urban areas.

\subsection{Uncontrolled Situation}

Under uncontrolled situation, the government's guiding and controlling role will be minimized. At this time, it is only limited by topographical factors. Market and capital are the dominant factors in urban development, and economic development has become the only driving force for urban expansion. At this time, the city will spread the pancake-style expansion, and the urban development model is more extensive. It is estimated that the construction land of the three cities of Changsha, Zhuzhou and Xiangtan will expand to the surrounding area. In addition to the conventional expansion to the surrounding area, Changsha may focus on the north-south direction along the Xiangjiang River in the future, connecting the northwest with Wangcheng, and connecting Zhuzhou to the south; And the administrative area restrictions, mainly to the north and Changsha into one, the development of the west and south is not much; in addition to developing westward and connecting with Xiangtan, Zhuzhou can choose to expand "along the port, along the river and along the mountain" to the northeast, south and east. In this process, many natural landscape resources, such as ecological green areas, green land, mountains, agricultural land and other natural land will be encroached (Figure 10(a)).

\subsection{General Control Situation}

Under general control situation, the three dominant factors of government, market and nature are relatively balanced. Under the constraints of natural factors, the three municipal governments (the three cities' overall planning) generally guide the development of urban construction land in the three cities. With the direction and speed of development, the market will be developed and constructed in an orderly manner under the guidance of the government. At this time, urban development will consider the limits of waters and basic farmland on urban development. The outward expansion of the city and the optimization of urban internal renewal will be carried out at the same time. However, in order to maintain the rapid development of their respective cities and enhance their economic strength, the three cities still occupy a dominant position in terms of outward expansion, and some ecological land around the city is still being occupied by Construction land.

It is expected that the plaques in the three cities of Changsha, Zhuzhou and 


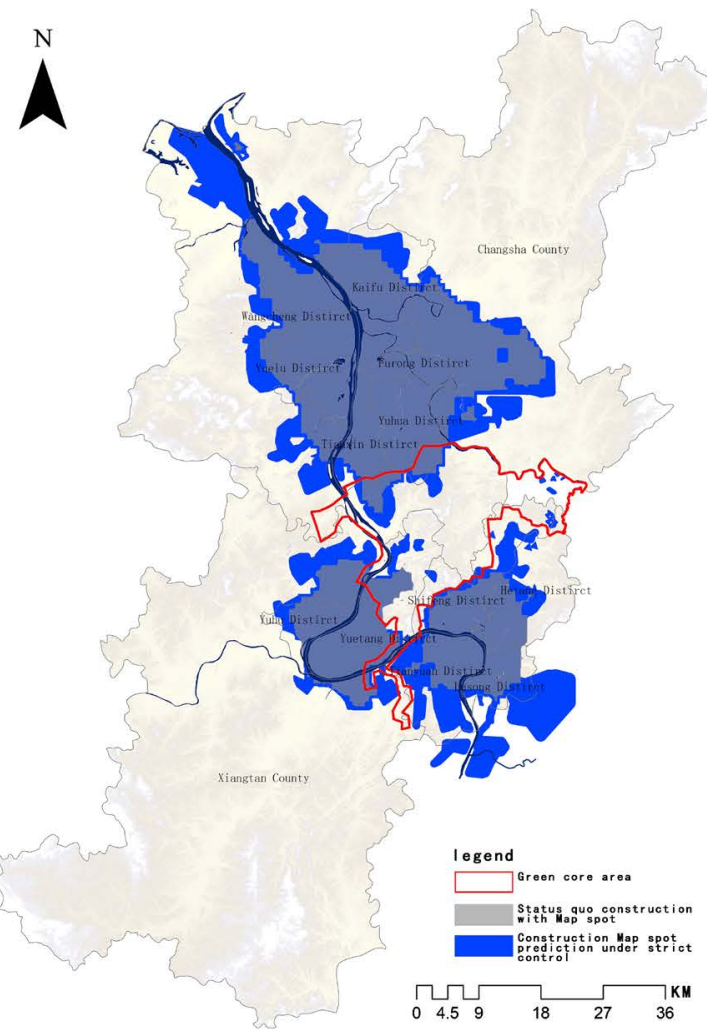

(a)

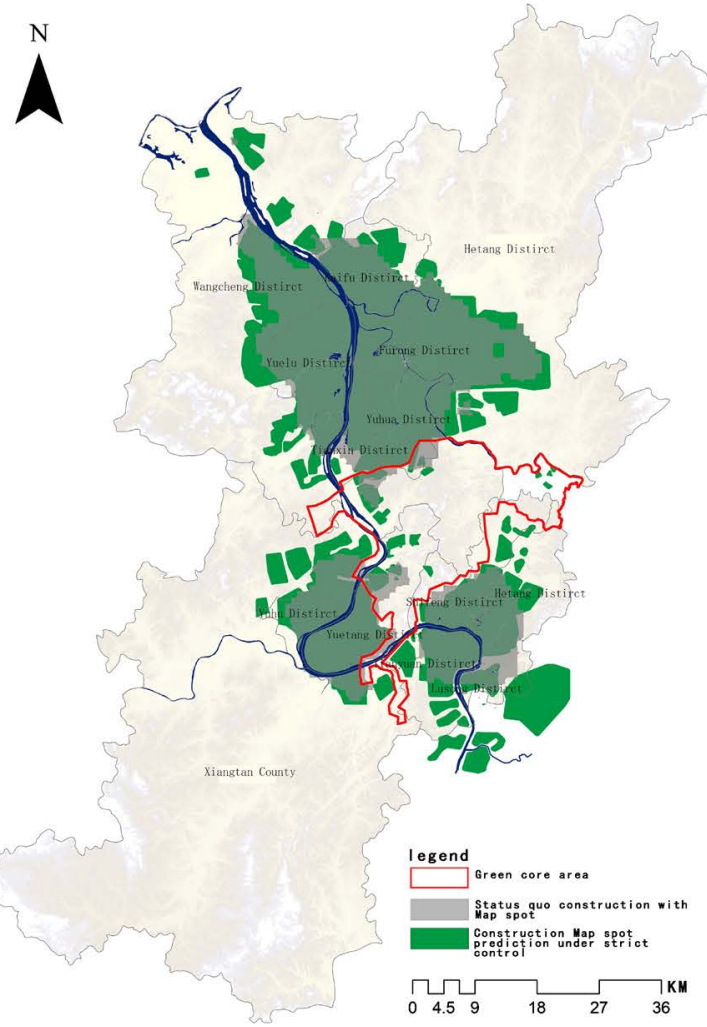

(b)

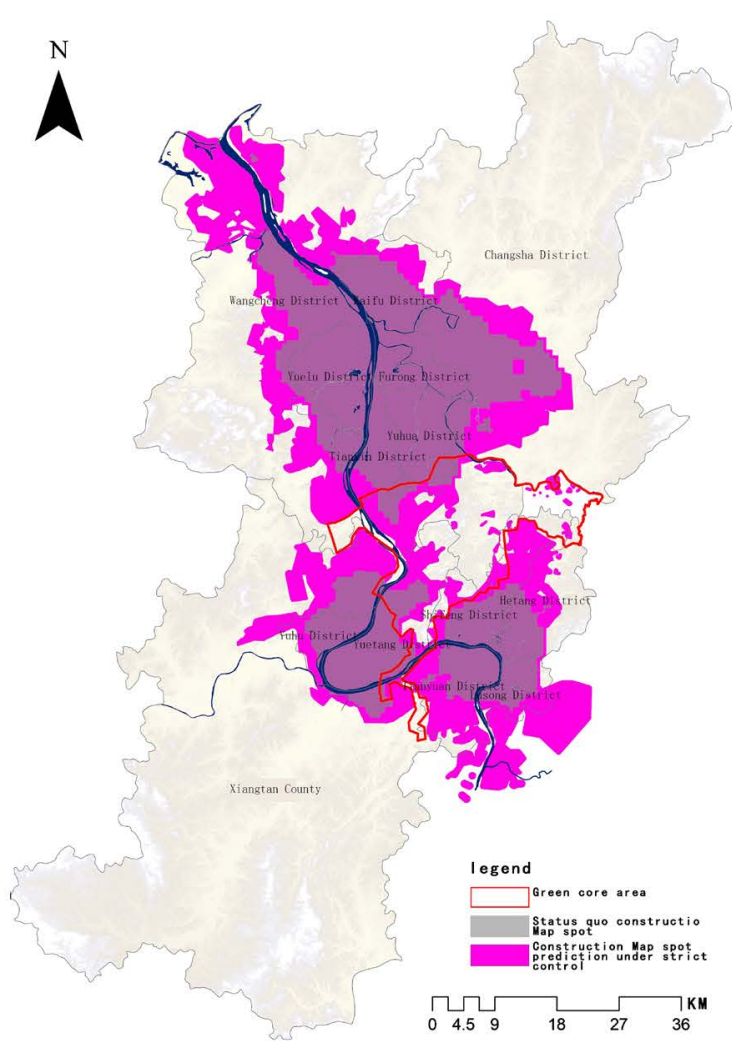

(c)

Figure 10. Prediction of construction land expansion under three control scenarios. ((a) Uncontrolled situation; (b) General control situation; (c) Strictly controlled situation). 
Xiangtan will be partially converged, and the development speed will be reduced. However, the overall development direction is similar to that under the unregulated conditions. The urban master plan compiled by the three cities of Changsha, Zhuzhou and Xiangtan under their respective development plans is more considered. With the development of their respective cities, the development trend of the three cities along the banks of the Xiangjiang River is obvious. Zhuzhou and Xiangtan are both built on the boundaries of administrative planning, leaving no green buffer. In the inner area of the green heart, especially along the boundary between the Xiangjiang River and Zhuzhou Xiangtan, it is highly eroded (Figure 10(b)).

\subsection{Strictly Controlled Situation}

Under Strictly controlled situation, the government, as the leading factor in urban development, strictly controls the quality and speed of urban development. The market is developed and constructed in strict accordance with the government's plan under the strong government constraints. In this process, urban development pays more attention to the protection of the ecological environment and natural resources. For the newly developed urban construction land, the expansion of the cake model is no longer used. Through strict control of urban intensive development, and attention to coordination with the surrounding environment of the city, focus on the upgrading and intensive transformation of existing construction land to improve the quality of urban development.

It is expected that under strict management and control conditions, cities will pay more attention to intensive development. The overall expansion of construction land in the three cities is relatively small, and the large amount of ecological land previously invaded will gradually be retired in the process of urban renewal and development, thus restoring the original Ecological attributes. Due to the serious development of the extensive expansion in Changsha, the area of urban construction land has been very large, and the future development is more to digest the stock of internal land, thus improving the quality of existing urban construction land. A small amount of new construction land should pay great attention to coordination with surrounding land. Zhuzhou Xiangtan is relatively more new construction land, through the expansion of multiple directions, gradually narrow the gap with Changsha, and stabilize the balance between the three cities. The three cities can form a good understanding, pay attention to the protection of the green environment, and do not invade the green land. Although the economy of Changsha, Zhuzhou and Xiangtan will be limited to a certain extent under the strict control situation, improving the quality of land use is the fundamental guarantee for the sustainable development of the three cities (Figure 10(c)).

\section{Conclusions}

1) From 1993 to 2019, the expansion speed of construction land in Chang- 
sha-Zhuzhou-Xiangtan metropolitan area shows a periodic fluctuation trend of "slow down, speed up, slow down, speed up and slow down", and the expansion direction of construction land correspondingly presents a periodic fluctuation trend of "centripetal, discrete, centripetal, discrete and centripetal". The spatial gravity of the metropolitan area shifts to the northwest, and the spatial form as a whole presents a "South-North" belt spread.

2) There are two completely different evolution trends in the landscape form of the inner and outer part of Changsha-Zhuzhou-Xiangtan metropolitan area. The pattern of the construction land patches in the outer part of the metropoli$\tan$ area is becoming more and more complicated, the number of patches is increasing, and the trend of fragmentation is obvious. The inner land patches are integrated with each other, and the connectivity is enhanced, showing the spatial intensive development. The two development trends coexist, but the outer landscape fragmentation trend is more prominent.

3) During the decade of 2006-2015, the three cities of Changsha, Zhuzhou and Xiangtan are developing towards each other, and the trend of space integration is the most obvious, and the green core area of urban agglomeration is severely eroded. Although the scale of spatial expansion of Changsha has always maintained an absolute advantage, Zhuzhou and Xiangtan have the most invasion to the green core region.

4) Analysis and comparison of three different spatial expansion scenarios in the metropolitan area, only through strict control and protection of the ecological barrier in the Changzhutan green core area, can we effectively avoid the spread of urban agglomerations, reduce diseases in big cities, and meet the future high-quality development needs of Changsha-Zhuzhou-Xiangtan urban agglomerations.

\section{Acknowledgements}

This research was funded by the Natural Science Foundation of China, grant number 51478470. The authors would like to thank NSCF.

\section{Conflicts of Interest}

The authors declare no conflicts of interest regarding the publication of this paper.

\section{References}

[1] Zhu, S.-J. (2012) Study on the Spatial Structure of Chang-Zhu-Tan Urban Agglomeration and Its Optimization. Central South University, Changsha.

[2] Zhou, G.-H., Tang, C.-L. and Zhu, X. (2002) A Study on Optimization of the Spatial Structure of Urban Agglomeration in Changsha-Zhuzhou-Xiangtan. Tropical Geography, 22, 330-334.

[3] Wang, L.-J., Zhou, K.-G. and Xu, C.-J. (2005) A Demonstrative Study on Travel Consumption Preference of Foreign Visitors to China. Geography and Geo-Information Science, 21, 78-103.

[4] Tang, F.-H., Chen, L.-L. and Zeng, Z.-W. (2010) Evolution Tends and Restructuring 
of Spatial Structure about Urban Agglomeration: By the Case of Chang-Zhu-Tan Agglomeration. Urban Studies, 17, 65-69.

[5] Elvidge, C.D. (1997) Mapping City Lights with Nighttime Data from the DMSP Operational Line Scan System. Photogrammetric Engineering and Remote Sensing, 63, 727-734.

[6] Elvidge, C.D., Baugh, K.E., Dietz, J.B., et al. (1999) Radiance Calibration of DMSP-OLS Low-Light Imaging Data of Human Settlements. Remote Sensing \& Environment, 68, 77-88. https://doi.org/10.1016/S0034-4257(98)00098-4

[7] Imhoff, M.L., Lawrence, W.T., Stutzer, D.C., et al. (1997) A Technique for Using Composite DMSP/OLS "City Lights" Satellite Data to Map Urban Area. Remote Sensing of Environment, 61, 361-370. https://doi.org/10.1016/S0034-4257(97)00046-1

[8] Huang, X., Schneider, A. and Friedl, M.A. (2016) Mapping Sub-Pixel Urban Expansion in China Using MODIS and DMSP/OLS Nighttime Lights. Remote Sensing of Environment, 175, 92-108. https://doi.org/10.1016/j.rse.2015.12.042

[9] Yang, Y., Huang, Q.-X. and Zhang, L.-L. (2015) The Spatial-Temporal Measurement on the Land Urbanization Level Using DMSP/OLS Nighttime Light Data-A Case Study of Bohai Rim. Economic Geography, 35, 141-148.

[10] Wang, Y.-Y., Xu, D. and Zhu, X.-G. (2010) The Spatio-Temporal Characteristics of the Urban and Town Construction Land Expansion in Jiangsu Province from DMSP-OLS Nighttime Images. Modern Urban Research, No. 2, 67-73.

[11] Zhou, Y., Smith, S.J., Elvidge, C.D., et al. (2014) A Cluster-Based Method to Map Urban Area from DMSP/OLS Nightlights. Remote Sensing of Environment, 147, 173-185. https://doi.org/10.1016/j.rse.2014.03.004

[12] Li, J.-G., He, C.-Y. and Shi, P.-J. (2007) The Use of Multisource Satellite and Geospatial Data to Study the Ecological Effects of Urbanization: A Case of the Urban Agglomerations in Bohai Rim. Journal of Remote Sensing, 11, 115-126.

[13] Shu, S., Yu, B.-L. and Wu, J.-P. (2011) Methods for Deriving Urban Built-Up Area Using Night-light Data: Assessment and Application. Remote Sensing Technology and Application, 26, 169-176.

[14] Liu, C.-X., Zhu, K.-W. and Li, Y.-C. (2017) Research on the Urban Expansion in Chengdu-Chongqing Economic Zone Based on DMSP/OLS Nighttime Light Data in Recent 20 Years. Journal of Chongqing Normal University (Natural Science), No. 6, 117-126.

[15] Wang, H.-R., Zheng, X.-Q. and Yuan, T. (2012) Overview of Researches Based on DMSP/OLS Nighttime Light Data. Progress in Geography, 10, 11-18.

[16] Yan, M. and Huang, J.-C. (2013) Review on the Research of Urban Spatial Expansion. Progress in Geography, 32, 1039-1050.

[17] Mi, X.-N., Bai, L.-Y. and Tan, X.-H. (2013) A New Method of Extracing Areas of Center City Regions Based on DMSP/OLS Data. Geo-Information Science, 15, 255-261. https://doi.org/10.3724/SP.J.1047.2013.001255

[18] Wu, J.G. (2007) Landscape Ecology: Pattern, Process Scale and Hierarchy. Higher Education Press, Beijing.

[19] Gangopadhyay, K. and Basu, B. (2010) City Size Distributions for India and China. Physica A Statistical Mechanics \& Its Applications, 388, 2682-2688. https://doi.org/10.1016/j.physa.2009.03.019

[20] Xu, J. (2016) Simulation Study of Dynamic Change of Spatial Land Use Pattern in Changsha-Zhuzhou-Xiangtan Urban Agglomeration Based on CLUE-S Model.

[21] Wang, H.-M. (2016) The Pattern Evolution of Ecological Land in Changsha-ZhuzhouXiangtan Core Area. 